\title{
Transmission capacity for dual-hop relaying in wireless ad hoc networks
}

\author{
Jaeyoung Lee ${ }^{1}$, Hyundong Shin², Joon Tae Kim³ and Jun Heo ${ }^{3 *}$
}

\begin{abstract}
To account for randomly distributed nodes in a wireless ad hoc network, the transmission capacity is defined as the number of successful transmissions taking place in the network per unit area under an outage constraint. In this paper, we analyze the transmission capacity for dual-hop relaying in a wireless ad hoc network in the presence of both cochannel interference and thermal noise, where interferers are spatially distributed following a Poisson distribution. Specifically, we first present the exact outage probability for amplify-and-forward and decode-andforward protocols in a Poisson field of interferers without neglecting noise at all nodes. We then derive the transmission capacity of such networks, which determines the maximum allowable density of transmitting nodes for each relay strategy at a specified outage probability and data rate. Numerical results demonstrate that the dualhop relaying is still beneficial in terms of the transmission capacity in wireless ad hoc Poisson networks.
\end{abstract}

Keywords: amplify-and-forward (AF), decode-and-forward (DF), dual-hop relay, interference, outage probability, Poisson network, transmission capacity

\section{Introduction}

Cooperative relay communication has recently drawn considerable attention to increase network coverage and reliability $[1,2]$. For a decode-and-forward (DF) strategy, a relay node decodes the received signal and transmits it after re-encoding, whereas the relay simply retransmits a scaled version of the received signal in amplify-and-forward (AF) mode [3,4]. Most of the previous work has focused on noise-limited fading environments, such as Rayleigh fading with additive white Gaussian noise (AWGN). While such work made great strides toward understanding the potential of relay networks, it dealt mainly with ideal configurations with no interference. However, network interference is inevitable in practical wireless networks due to spectral reuse. Motivated by this, relay networks have been recently studied in the presence of cochannel interference [5-7]. In Krikidis et al. [5], the asymptotic performance was analyzed under the interference scenario only at relays. The fixed-gain AF relaying in an interference network was investigated in Zhong et al. [6], where interference at the relay and

\footnotetext{
* Correspondence: junheo@korea.ac.kr

${ }^{1}$ School of Electrical Engineering, Korea University, 5-1 Anam-dong, Sungbukgu, Seoul, Republic of Korea
}

Full list of author information is available at the end of the article thermal noise at the destination were ignored for analytical tractability. The exact outage probability for DF relaying was further derived in $\mathrm{Si}$ et al. [7] accounting for multiple interferers and noise at both the relay and the destination. In all these work, the locations of network nodes are deterministic-without spatial randomness.

To treat the capacity of a decentralized ad hoc wireless network, the transport capacity, defined as the product of the end-to-end sum throughput and distance, has been introduced due to the difficulty in determining the capacity region of a large ad hoc network [8]. More recently, using the stochastic geometry framework, the transmission capacity has been proposed as the maximum density of active transmitting nodes per unit area to satisfy an outage constraint at a given data rate when interferers are randomly scattered and uncoordinated [9]. For a variety of scenarios, this notion of transmission capacity has been used successfully to characterize the physical layer on the ad hoc network [10-14]. A new metric akin to the transmission capacity, called the random access transport capacity, has been also developed for the end-to-end throughput in multi-hop transmission over some distance [15].

In this paper, we consider dual-hop relaying with DF and AF protocols in a wireless ad hoc network in the 
presence of both interference and noise. Each interfering node in the network independently transmits data and is randomly distributed in a Poisson law over a plane. The motivation behind imposing Poisson interference and noise is threefold: (i) many previous works neglected either interference or noise in analysis of a relay network; (ii) previous work on relay networks with interference considered only a limited number of interferers (iii) randomly distributed nodes(i.e., interferers) allowing a Poisson distribution are suitable for realistic communication model. To the best of our knowledge, relay network to qualify interference using stochastic geometry has not been addressed before. Hence, we analyze exact outage probability of a dual-hop relay with both noise and interference in a wireless ad hoc network. Unlike previous work in a relay network, when the node locations are distributed as a Poisson point process (PPP), we need to analyze relay networks using a metric for decentralized wireless network, termed transmission capacity. Furthermore, as transmission capacity has considered single-hop transmission without noise, we focus on the transmission capacity of dual-hop relay with noise and specific relaying protocols. It is worth of finding maximum successful transmitting nodes per unit area to satisfy outage probability and data rate from transmission capacity of dual-hop relay. Although both noise and interference with Poisson distribution are considered, it is noted that dual-hop relay is still beneficial in terms of transmission capacity.

The remainder of the paper is organized as follows. Section 2 presents the system model based on location of nodes, channel models, and distribution of interference. We derive outage probability analysis of DF and AF strategies in Section 3. Based on the outage probability of dual-hop relay, transmission capacity is derived in Section 4. Section 5 compares simulation results with analytical results. Finally, Section 6 concludes the paper.

\section{System model}

We consider a wireless ad hoc network consisting of a source, a relay, and a destination with no direct sourceto-destination link. All nodes have a single antenna operating in a common frequency band and are in halfduplex mode.

\subsection{Node locations}

The sets of nodes in a relay network are denoted as $\mathcal{S}:=\left\{S x_{n}, n \in \mathbb{N}\right\}$ for sources, $\mathcal{R}:=\left\{R x_{n}, n \in \mathbb{N}\right\}$ for relays, and $\mathcal{D}:=\left\{D x_{n}, n \in \mathbb{N}\right\}$ for destinations. Now, allow the set of nodes to be divided into two different pairs for each hop transmission: $\left(S x_{n}, R x_{n}\right)$ and $\left(R x_{n}\right.$, $\left.D x_{n}\right)$, where the distance between two nodes in a pair is $d_{S R}$ and $d_{R D}$, respectively. In a dual-hop relay network, sources and relays are also interferers in each hop. In particular, all nodes independently transmit and distribute their locations following a Poisson distribution. Let the location of $S x_{n}$ be $S_{n}, R x_{n}$ be $R_{n}$, and $D x_{n}$ be $D_{n}$. The source set $\Phi_{\mathcal{S}}:=\left\{S_{n}\right\}$ generating interference at the first time slot is modeled as a homogeneous PPP on the plane of intensity $\lambda_{1}$. Since $R_{n}$ is randomly located at a fixed distance $d_{S R}$ from $S_{n}$, the set $\Upsilon_{\mathcal{R}}:=\left\{R_{n}\right\}$ also follows a homogeneous PPP on a two-dimensional plane with intensity $\lambda_{2}$. As destination node $D_{n}$ is placed at $d_{R D}$ away from the relay node $R_{n}, \Psi_{\mathcal{D}}:=\left\{D_{n}\right\}$ is also a homogeneous PPP with intensity $\lambda_{3}$.

In this paper, we consider reference source $S x_{0}$, reference relay $R x_{0}$, and reference destination $D x_{0}$ in a decentralized wireless network that transmits desirable data in the $\Phi_{S}, \Upsilon_{R}$, and $\Psi_{D}$. According to each hop transmission, reference nodes can be bounded as $\left(S x_{0}\right.$, $\left.R x_{0}\right)$ and $\left(R x_{0}, D x_{0}\right)$. For the $\left(S x_{0}, R x_{0}\right)$ pair in the first hop, the relay node $R x_{0}$ is located at the origin and source node $S x_{0}$ is placed at $d_{S R}$ meters away from the relay node $R x_{0}$. For the $\left(R x_{0}, D x_{0}\right)$ pair in the second hop, we place the relay node $R x_{0} d_{R D}$ meters away from the destination node $D x_{0}$, where $D x_{0}$ is located at the origin.

\subsection{Channel model}

In this paper, we consider a channel model with smallscale fading and large-scale path loss for a practical situation. Therefore, the square of instantaneous channel response between any pair of nodes located at $x_{1}$ and $x_{2}$ can be rewritten as

$$
\left|h_{12}\right|^{2}=F_{12} \cdot l\left(\left\|x_{1}-x_{2}\right\|\right),
$$

where $F_{12}$ captures the small-scale fading which obeys a Rayleigh fading model and $l\left(\left\|x_{1}-x_{2}\right\|\right)=\left\|x_{1}-x_{2}\right\|^{-\alpha}$ characterizes the effect of large-scale path loss following the power law with path loss exponent $\alpha$. We assume that channel responses for all nodes are independent and quasi-static.

\subsection{Distribution of aggregate interference}

In this section, we characterize the total interference power measured at the origin, as follows

$$
\mathrm{I}=\sum_{i \in \Phi}\left|\mathrm{h}_{i}\right|^{2} P_{i}
$$

where $\mathrm{h}_{i}$ is a instantaneous channel response of interferer $i, \Phi$ is a homogenous PPP with intensity $\lambda$, and $P_{i}$ is interference power.

The moment generating function $(M G F)$ of total interference power using method in Haenggi and Ganti [16], denoted by $\phi_{I}(s) \triangleq \bullet\left\{e^{-s I}\right\}$, can be written as 


$$
\phi_{I}(s)=\exp \left(-\lambda c_{d} \Gamma(1+\delta) \gamma(1-\delta, r) P_{i}^{\delta} s^{\delta}\right)
$$

where $\lambda$ is intensity of interferers, $c_{d}$ is the volume of $d$-dimensional unit ball, $r$ is the radius of finite area located interferers, and $\delta \triangleq d / \alpha$. Also, $\Gamma(x)$ is the Gamma function $\int_{0}^{\infty} t^{x-1} e^{-t} d t$ and $\gamma(s, x)$ is the lower incomplete gamma function $\int_{0}^{x} t^{s-1} e^{-t} d t$.

\section{Outage performance analysis}

\subsection{Decode-and-forward relaying}

We consider a dual-hop relay system that consists of reference source, relay, and destination nodes in a Poisson network as shown in Figure 1. Other nodes except the reference nodes are regarded as interferers. We consider interferers in a finite area with specific radius, because nodes located far from a receiver cannot be an interferer. In the first hop, relay nodes receive signal transmitted from source nodes and the received signal $y_{R}$ at a reference relay node $R x_{0}$ can be expressed as

$$
\gamma_{R}=\mathrm{h}_{S R} \mathrm{~s}_{0}+\sum_{i \in \Phi_{S}} \mathrm{~g}_{i} \mathrm{~s}_{i}+\mathrm{n}_{R}
$$

where $\mathrm{s}_{0}$ is the transmitted symbol with $E\left[\left|\mathrm{~s}_{0}\right|^{2}\right]=P_{S}$, $\mathrm{h}_{S R}$ is the channel between the reference source and the reference relay, and $\mathrm{n}_{R}$ is an additive white Gaussian noise with an average power of $\sigma_{R}^{2}$. In addition, the relay node $R x_{0}$ of the reference pair is affected by its interferes $s_{i}$ located in a homogenous PPP $\Phi_{S}$. Average transmit power of an interferer is $E\left[\left|\mathrm{~s}_{i}\right|^{2}\right]=P_{i R}$, and $\left\{\mathrm{g}_{i}\right\}_{i \in \Phi_{S}}$ are the channels from the interferer $i$ to the reference relay $R x_{0}$. Thus, the received signal to interference-plusnoise ratio (SINR) at the relay can be written as

$$
\gamma_{R}^{\mathrm{DF}}=\frac{P_{S}\left|\mathrm{~h}_{S R}\right|^{2}}{\sum_{i \in \Phi_{S}}\left|\mathrm{~g}_{i}\right|^{2} P_{i R}+\sigma_{R}^{2}} .
$$

On the other hand, at the reference destination $D x_{0}$, a transformed copy of the received signal at the relay, $\mathcal{T}\left(y_{R}\right)$, is then transmitted to the destination node, which suffers from the other relay's transmission $\left\{\mathrm{w}_{j}\right\}_{j \in \Upsilon_{R}}$ located in a homogeneous PPP $\Upsilon_{R}$, each average transmitting power of an interferer $j$ is $E\left[\left|\mathrm{w}_{j}\right|^{2}\right]=P_{j D}$. Thus, the received signal at the destination $D x_{0}$ is given by

$$
y_{D}=\mathrm{h}_{R D} \mathcal{T}\left(y_{R}\right)+\sum_{j \in \Upsilon_{R}} \mathrm{I}_{j} \mathrm{w}_{j}+\mathrm{n}_{D}
$$

where $\mathrm{h}_{R D}$ is the channel between the reference relay and the reference destination, $\mathrm{n}_{D}$ is an additive white Gaussian noise with an average power of $\sigma_{D}^{2}$, and $\left\{l_{j}\right\}_{j \in \Phi}$ are the channels from the interferer $j$ to the reference destination. In DF, the relay fully decodes the message $s_{0}$ based on the received signal, $y_{R}$, and then transmits the re-encoded message $\mathcal{T}\left(y_{R}\right)=\mathrm{s}_{r}$ with a power of $P_{R}$ to the destination terminal. Thus, the received SINR at the destination can be written as

$$
\gamma_{D}^{\mathrm{DF}}=\frac{P_{R}\left|\mathrm{~h}_{R D}\right|^{2}}{\sum_{j \in \Upsilon_{R}}\left|\mathrm{I}_{i}\right|^{2} P_{j D}+\sigma_{D}^{2}} .
$$

First, to compute cumulative density function $(C D F)$ and probability density function $(P D F)$ of $\gamma_{R}^{\text {DF }}$, we define $\mathrm{P} \sim P_{S}\left|\mathrm{~h}_{S R}\right|^{2}$ and $\mathrm{Q} \sim \sum_{\Phi_{S}}\left|\mathrm{~g}_{i}\right|^{2} P_{i_{R}}$. Then, the $C D F$ and $P D F$ of $\mathrm{P}$ can be written as

$$
\begin{array}{ll}
F_{\mathrm{P}}(p)=1-\exp \left(-\frac{d_{S R}^{\alpha}}{P_{S}} p\right), & p \geq 0 \\
p_{\mathrm{P}}(p)=\frac{d_{S R}^{\alpha}}{P_{S}} \exp \left(-\frac{d_{S R}^{\alpha}}{P_{S}} p\right), & p \geq 0 .
\end{array}
$$

where $d_{S R}$ is distance between reference source and reference relay and $\alpha$ is path loss exponent. As the main physical range of interest for path loss exponent in wireless networks is $2<\alpha<5$, we consider path loss exponent $\alpha=4$ for a relatively lossy environment in an urban area. From (3), the $M G F$ of total interference power $\mathrm{Q} \sim \sum_{\Phi_{S}}\left|\mathrm{~g}_{i}\right|^{2} P_{i R}$ with two-dimensional plane $\left(\delta=\frac{1}{2}\right.$ case and $\left.c_{d}=\pi\right)$ can be written as

$$
\phi_{Q}(s)=\exp \left(-\lambda_{1} \pi \Gamma\left(\frac{3}{2}\right) \gamma\left(\frac{1}{2}, x\right) \sqrt{P_{i R}} s \frac{1}{2}\right)=\exp \left(-\frac{\lambda_{1} \pi^{2} \operatorname{erf}\left(\sqrt{r_{1}}\right) \sqrt{P_{i R}}}{2} \frac{1}{2}\right)
$$

where $\Gamma\left(\frac{3}{2}\right)=\frac{\sqrt{\pi}}{2}$ and $\gamma\left(\frac{1}{2}, x\right)=\sqrt{\pi} \operatorname{erf}(\sqrt{x})$ with error function $\operatorname{erf}(x) \triangleq \frac{2}{\sqrt{\pi}} \int_{0}^{x} e^{-t^{2}} d t$. The $C D F$ and PDF of Q from (9) using the Levy distribution [16] with $c=\frac{\lambda_{1}^{2} \pi^{4}\left(\operatorname{erf}\left(\sqrt{r_{1}}\right)\right)^{2} P_{i R}}{8}$ and $M=0$ can be expressed as

$$
\begin{aligned}
& F_{Q}(q)=1-\operatorname{erf}\left(\frac{\pi^{2} \operatorname{erf}\left(\sqrt{r_{1}}\right) \lambda_{1} P_{i R}^{\frac{1}{2}}}{4 \sqrt{q}}\right), \quad q \geq 0 \\
& p_{Q}(q)=\frac{\operatorname{erf}\left(\sqrt{r_{1}}\right) \lambda_{1} P_{i R}^{\frac{1}{2}}}{4}\left(\frac{\pi}{q}\right)^{3 / 2} \exp \left(-\frac{\left[\operatorname{erf}\left(\sqrt{r_{1}}\right)\right]^{2} \pi^{4} \lambda_{1}^{2} P_{i R}}{16 q}\right), \quad q \geq 0
\end{aligned}
$$

where $\lambda_{1}$ is intensity of source nodes and $r_{1}$ denotes the radius of interference area.

Theorem 1 ( $C D F$ and $P D F$ ) The CDF and PDF of received SINR at the relay $\mathrm{X} \sim \gamma_{R}^{\mathrm{DF}}$ are given by

$$
\begin{aligned}
F_{\mathrm{X}}(x)= & -\exp \left[-\left(\frac{d_{S R}^{\alpha} \sigma_{R}^{2}}{P_{S}} x+\frac{\operatorname{erf}\left(\sqrt{r_{1}}\right) \pi^{2} \lambda_{1}}{2} \sqrt{\left.\left.\frac{d_{S R}^{\alpha} P_{i R}}{P_{S}} x\right)\right],} \quad x \geq 0\right.\right. \\
p_{X}(x)= & \left(\frac{d_{S R}^{\alpha} \sigma_{R}^{2}}{P_{S}}+\frac{\operatorname{erf}\left(\sqrt{r_{1}}\right) \pi^{2} \lambda_{1} \sqrt{d_{S R}^{\alpha} P_{i R}}}{4 \sqrt{P_{S} x}}\right) \\
& \times \exp \left[-\left(\frac{d_{S R}^{\alpha} \sigma_{R}^{2}}{P_{S}} x+\frac{\operatorname{erf}\left(\sqrt{r_{1}}\right) \pi^{2} \lambda_{1}}{2} \sqrt{\frac{d_{S R}^{\alpha} P_{i R}}{P_{S}} x}\right)\right], \quad x \geq 0
\end{aligned}
$$




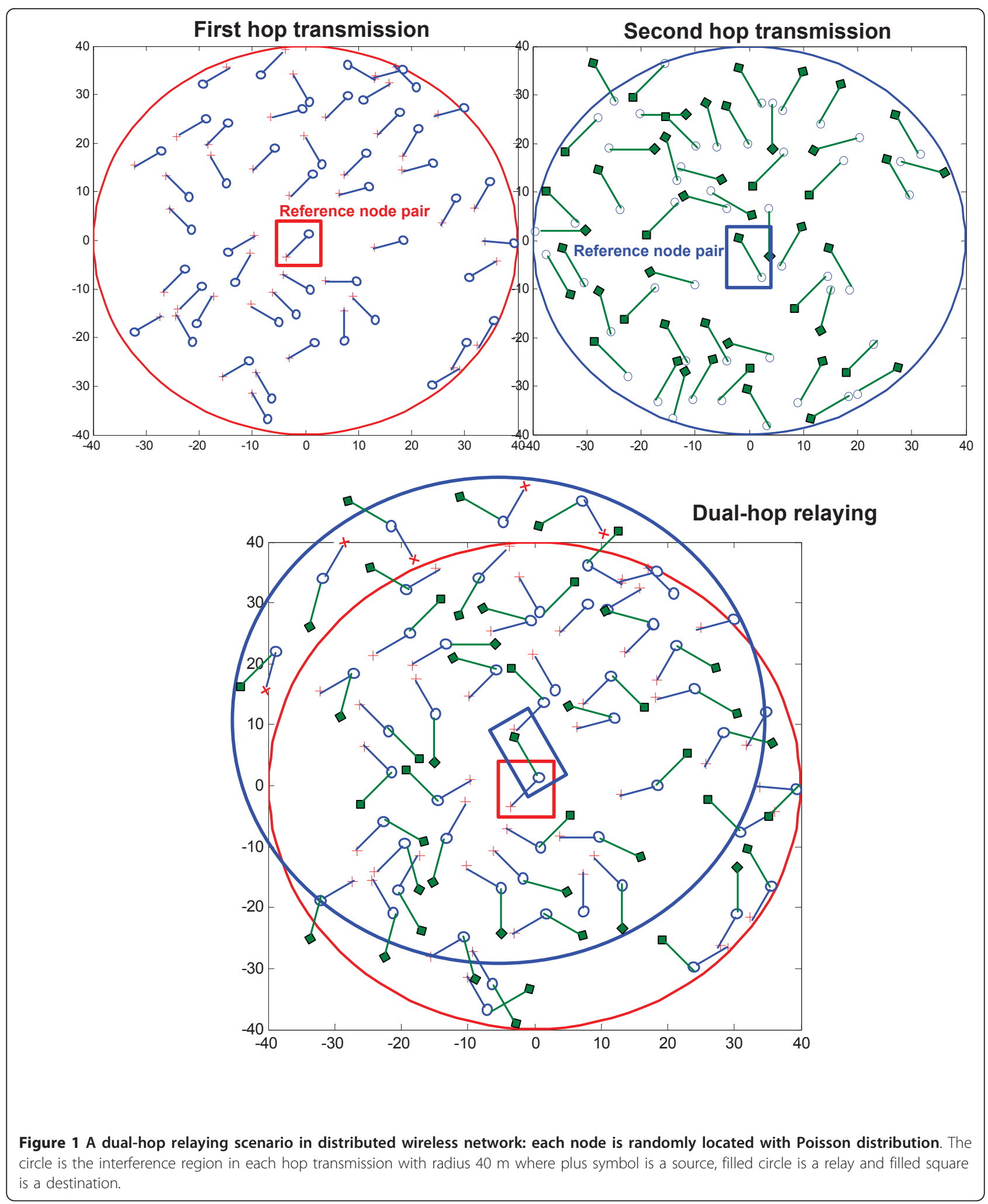


Proof See Appendix A

Corollary 1 Using the same method, the CDF and $P D F$ of the received SINR at the destination $\mathrm{Y} \sim \gamma_{D}^{\mathrm{DF}}$ can be written as

$$
\begin{aligned}
F_{\mathrm{Y}}(y)= & -\exp \left[-\left(\frac{d_{R D}^{\alpha} \sigma_{D}^{2}}{P_{R}} y+\frac{\operatorname{erf}\left(\sqrt{r_{2}}\right) \pi^{2} \lambda_{2}}{2} \sqrt{\frac{d_{R D}^{\alpha} P_{i D}}{P_{R}} \gamma}\right)\right], \quad y \geq 0 \\
p_{\mathrm{Y}}(y)= & \left(\frac{d_{R D}^{\alpha} \sigma_{D}^{2}}{P_{R}}+\frac{\operatorname{erf}\left(\sqrt{r_{2}}\right) \pi^{2} \lambda_{2} d_{R D}^{\alpha} P_{i D}}{\sqrt{4 P_{R} \gamma}}\right) \\
& \times \exp \left[-\left(\frac{d_{R D}^{\alpha} \sigma_{D}^{2}}{P_{R}} \gamma+\frac{\operatorname{erf}\left(\sqrt{r_{2}}\right) \pi^{2} \lambda_{2}}{2} \sqrt{\frac{d_{R D}^{\alpha} P_{i D}}{P_{R}} \gamma}\right)\right], \quad y \geq 0
\end{aligned}
$$

where $d_{R D}$ is the distance between relay and destination and $r_{2}$ is radius of finite area with intensity of interferers $\lambda_{2}$.

The end-to-end SINR of dual-hop DF relaying $\gamma_{\mathrm{eq}}$ can be written as

$$
\gamma_{\mathrm{eq}}-\min \left[\gamma_{R}^{\mathrm{DF}}, \gamma_{D}^{\mathrm{DF}}\right]
$$

Then, the outage probability can be represented as

$$
P_{\text {out }}^{\mathrm{DF}}(R)=\bullet\left\{\frac{1}{2} \log _{2}\left(1+\gamma_{\mathrm{eq}}\right)<R\right\}
$$

where $R$ is end-to-end spectral efficiency.

Finally, the outage probability of DF relaying can be expressed as

$$
\begin{aligned}
P_{\mathrm{out}}^{\mathrm{DF}}(\beta)= & F_{\mathrm{X}}(\beta)+F_{\mathrm{Y}}(\beta)-F_{\mathrm{X}}(\beta) F_{\mathrm{Y}}(\beta) \\
= & 1-\exp \left[-\left\{\left(\frac{d_{S D}^{\alpha} \sigma_{R}^{2}}{P_{S}}+\frac{d_{R D}^{\alpha} \sigma_{D}^{2}}{P_{R}}\right) \beta\right.\right. \\
& \left.\left.+\frac{\pi^{2}}{2}\left(\operatorname{erf}\left(\sqrt{r_{1}}\right) \lambda_{1} \sqrt{\frac{d_{S R}^{\alpha} P_{i R}}{P_{S}}}+\operatorname{erf}\left(\sqrt{r_{2}}\right) \lambda_{2} \sqrt{\frac{d_{R D}^{\alpha} P_{i D}}{P_{R}}}\right) \sqrt{\beta}\right\}\right], \quad \beta \geq 0
\end{aligned}
$$

where $\beta$ is target $\operatorname{SINR} \beta=2^{2 R}-1$

\subsection{Amplify-and-forward relaying}

In the first hop, the received signal at the reference relay of AF relaying is identical to (4). The received signal of AF relaying at the destination can be written as

$$
y_{D}=\mathrm{h}_{R D} G y_{R}+\sum_{j \in \Upsilon_{R}} \mathrm{I}_{j} \mathrm{w}_{j}+\mathrm{n}_{D}
$$

where $\mathrm{h}_{R D}$ is the channel between the reference relay and the reference destination, $\mathrm{n}_{D}$ is an additive white Gaussian noise with an average power of $\sigma_{D}^{2}$ and $\left\{l_{j}\right\}_{j \in} \gamma$ are the channels from the interferer $j$ to the reference destination. The amplification factor $G$ of AF relaying with interference [5] can be expressed as

$$
\mathrm{G}=\sqrt{\frac{P_{R}}{\left\|\mathrm{y}_{R}\right\|^{2}}}=\sqrt{\frac{P_{R}}{P_{S}\left|\mathrm{~h}_{S R}\right|^{2}+\sum_{i \in \Phi_{S}}\left|\mathrm{~g}_{i}\right|^{2} P_{i R}+\sigma_{R}^{2}}} .
$$

Combining (4) and (17), $y_{D}$ can be rewritten as

$$
\begin{aligned}
& \mathrm{y}_{D}=\mathrm{h}_{R D} \mathrm{G}\left(\mathrm{h}_{S R} \mathrm{~s}_{0}+\sum_{i \in \Phi s} \mathrm{~g}_{i} \mathrm{~s}_{i}+\mathrm{n}_{R}\right)+\sum_{j \in \Upsilon_{R}} \mathrm{I}_{j} \mathrm{w}_{j}+\mathrm{n}_{D}(19) \\
& =\mathrm{h}_{S R} \mathrm{~h}_{R D} \mathrm{Gs}_{0}+\mathrm{h}_{R D} \mathrm{G} \sum_{i \in \Phi s} \mathrm{~g}_{i} \mathrm{~s}_{i}+\sum_{i \in \Upsilon_{R}} \mathrm{I}_{j} w_{j}+\mathrm{h}_{R D} \mathrm{Gn}_{R}+(2 \mathcal{O}(\mathcal{P})
\end{aligned}
$$

End-to-end SINR at the destination of AF relaying can be given by

$$
\gamma_{D}^{\mathrm{AF}}=\frac{\left|\mathrm{h}_{S R}\right|^{2}\left|\mathrm{~h}_{R D}\right|^{2} \mathrm{G}^{2} P_{S}}{\left|\mathrm{~h}_{R D}\right|^{2} \mathrm{G}^{2} \sum_{i \in \Phi s}\left|\mathrm{~g}_{i}\right|^{2} P_{i R}+\sum_{j \in \Upsilon_{R}}\left|\mathrm{I}_{j}\right|^{2} P_{i D}+\left|\mathrm{h}_{R D}\right|^{2} \mathrm{G}^{2} \sigma_{R}^{2}+\sigma_{D}^{2}} .
$$

We define new random variables $R$ and $S$, and we use the random variables $\mathrm{P}$ and $\mathrm{Q}$ in the previous section as follows:

$$
P \sim\left|\mathrm{h}_{S R}\right|^{2} P_{S}, \quad \mathrm{Q} \sim \sum_{i \in \Phi}\left|\mathrm{g}_{i}\right|^{2} P_{i R}, \quad \mathrm{R} \sim\left|\mathrm{h}_{R D}\right|^{2} P_{R}, \quad \mathrm{~S} \sim \sum_{j \in Y_{R}}\left|\mathrm{I}_{j}\right|^{2} P_{i D} .
$$

We rearrange the end-to-end SINR (21) to make the Macdonald random variable form in Barua et al. [4] as

$$
\begin{aligned}
\gamma_{D}^{\mathrm{AF}} & =\frac{\mathrm{PR}}{\mathrm{SP}+a \mathrm{~S}+\mathrm{RQ}+b \mathrm{Q}+a \mathrm{R}+b \mathrm{P}+a b+\mathrm{QS}} \\
& =\frac{\mathrm{PR}}{\mathrm{P}(\mathrm{R}+b)+\mathrm{S}(\mathrm{P}+a)+(\mathrm{P}+a)(\mathrm{R}+b)-\mathrm{PR}+\mathrm{QS}} \\
& =\frac{\mathrm{PR}}{\mathrm{S}(\mathrm{P}+\mathrm{Q}+a)+(\mathrm{P}+\mathrm{Q}+a)(\mathrm{R}+b)-\mathrm{PR}} \\
& =\frac{\mathrm{PR}}{(\mathrm{P}+\mathrm{Q}+a)(\mathrm{R}+\mathrm{S}+b)-\mathrm{PR}}=\frac{1}{\left(\frac{\mathrm{P}+\mathrm{Q}+a}{\mathrm{P}}\right)\left(\frac{\mathrm{R}+\mathrm{S}+b}{\mathrm{R}}\right)-1} \\
& =\frac{1}{\frac{\mathrm{Q}+a}{\mathrm{P}}+\frac{\mathrm{S}+b}{\mathrm{R}}+\frac{(\mathrm{Q}+a)(\mathrm{S}+b)}{\mathrm{PR}}}=\frac{\frac{\mathrm{P}+a}{\mathrm{P}} \cdot \frac{\mathrm{R}}{\mathrm{S}+b}}{\frac{\mathrm{Q}+a}{\mathrm{~S}+b}+1}
\end{aligned}
$$

where $a=\sigma_{R}^{2}$ and $b=\sigma_{D}^{2}$.

Hence, end-to-end SINR of AF relaying can be written as

$$
\gamma_{D}^{\mathrm{AF}}=\frac{\frac{\left|\mathrm{h}_{S R}\right|^{2} P_{S}}{\sum_{i \in \Phi_{S}}\left|\mathrm{~g}_{i}\right|^{2} P_{i R}+\sigma_{R}^{2}} \cdot \frac{\left|\mathrm{h}_{R D}\right|^{2} P_{R}}{\sum_{j \in \Upsilon_{S}}\left|\mathrm{I}_{j}\right|^{2} P_{i D}+\sigma_{D}^{2}}}{\frac{\left|\mathrm{h}_{S R}\right|^{2} P_{S}}{\sum_{i \in \Phi_{S}}\left|\mathrm{~g}_{i}\right|^{2} P_{i R}+\sigma_{R}^{2}}+\frac{\left|\mathrm{h}_{R D}\right|^{2} P_{R}}{\sum_{j \in \Upsilon_{R}}\left|\mathrm{I}_{\mathrm{j}}\right|^{2} P_{i D}+\sigma_{D}^{2}}+1=\frac{\mathrm{XY}}{\mathrm{X}+\mathrm{Y}+1}}
$$

where

$$
\mathrm{X} \sim \frac{\left|\mathrm{h}_{S R}\right|^{2} P_{S}}{\sum_{i \in \Phi_{S}}\left|\mathrm{~g}_{i}\right|^{2} P_{i R}+\sigma_{R}^{2}}, \quad \mathrm{Y} \sim \frac{\left|\mathrm{h}_{R D}\right|^{2} P_{R}}{\sum_{j \in \Upsilon_{R}}\left|\mathrm{I}_{j}\right|^{2} P_{i D}+\sigma_{D}^{2}}
$$

The random variable $\mathrm{X}$ and $\mathrm{Y}$ were defined in $\mathrm{DF}$ relaying, and the $P D F$ and $C D F$ of them are the same as in Theorem 1 and Corollary 1.

Theorem 2 (Outage probability of AF relaying) From the PDF and CDF of $\mathrm{X}$ and $Y$, we can compute outage probability of $A F$ relaying as 


$$
\begin{aligned}
P_{\mathrm{out}}^{\mathrm{AF}}(\beta)= & -B \int_{0}^{\infty} \exp \left[-\left(A\left(\frac{\beta(u+\beta+1)}{u}\right)+B(u+\beta)\right.\right. \\
& \left.\left.+C \sqrt{\frac{\beta(u+\beta+1)}{u}}+D \sqrt{u+\beta}\right)\right] d u \\
& -\frac{D}{2} \int_{0}^{\infty} \frac{1}{\sqrt{u+\beta}} \exp \left[-\left(A\left(\frac{\beta(u+\beta+1)}{u}\right)+B(u+\beta)\right.\right. \\
& \left.\left.+C \sqrt{\frac{\beta(u+\beta+1)}{u}}+D \sqrt{u+\beta}\right)\right] d u
\end{aligned}
$$

where

$$
\begin{aligned}
& \beta=2^{2 R}-1, \quad A=\frac{d_{S R}^{\alpha} \sigma_{R}^{2}}{P_{S}}, \quad B=\frac{d_{R D}^{\alpha} \sigma_{D}^{2}}{P_{R}}, \\
& C=\frac{\operatorname{erf}\left(\sqrt{r_{1}}\right) \pi^{2} \lambda_{1}}{2} \sqrt{\frac{d_{S R}^{\alpha} P_{i R}}{P_{S}}}, \quad D=\frac{\operatorname{erf}\left(\sqrt{r_{2}}\right) \pi^{2} \lambda_{2}}{2} \sqrt{\frac{d_{R D}^{\alpha} P_{i D}}{P_{R}} .}
\end{aligned}
$$

\section{Proof See Appendix B}

\section{Transmission capacity analysis}

In this section, we compute transmission capacity $C(\epsilon)$ based on outage probability of a dual-hop relay. Transmission capacity was defined as the maximum density of the transmitting node to satisfy outage probability and data rate on medium access control (MAC) layer performance. That is, it is efficient to find the maximum available transmitting source and relay nodes to satisfy a given outage probability and data rate (i.e., Quality of Service (QoS)) from the transmission capacity of a dualhop relay. As transmission capacity in Weber et al. [13] considers single-hop transmission without noise, we focus on the transmission capacity of a dual-hop relay with noise and specific relaying protocols. We assume that the sets of $\Phi_{S}, \Upsilon_{R}$, and $\Psi_{D}$ are homogeneous PPPs on the two-dimensional plane with the same intensity $\lambda$ due to the random translation invariance property of PPP [17].

\subsection{Direct transmission}

Transmission capacity of a single hop $C(\epsilon)[13,14]$ is defined as

$$
C(\varepsilon)=R q^{-1}(\varepsilon)(1-\varepsilon) \quad\left(\mathrm{bps} / \mathrm{Hz} / \mathrm{m}^{2}\right)
$$

where $\epsilon$ is outage probability for target $\operatorname{SINR} \beta=2^{R}$ 1 with spectral efficiency $R, q^{-1}(\epsilon)$ is the spatial intensity of attempted transmission associated with outage probability $\epsilon$ and it is always greater than 0 . The transmission capacity $C(\epsilon)$ is thinned by the probability of success $1-\epsilon$.

We derive transmission capacity of direct (single hop) transmission with noise in this section. For computing outage probability of direct transmission between source and destination, the received SINR at the destination is represented as

$$
\gamma_{D}=\frac{P_{S}\left|\mathrm{~h}_{S D}\right|^{2}}{\sum_{k \in \Phi_{S}}\left|\mathrm{f}_{k}\right|^{2} P_{k D}+\sigma_{D}^{2}}
$$

where $\mathrm{h}_{S D}$ is channel between source and destination, $\left\{\mathrm{f}_{k}\right\}_{k \in \Phi s}$ are the channels from the interferer $k$ to the reference destination, and $P_{k D}$ is each average transmitting power of interferer $k$ to destination. Similar to Theorem 1 , the outage probability of direct transmission between source and destination can be given by

$$
P_{\text {out }}^{D T}(\beta)=1-\exp \left[-\left(\frac{d_{S D}^{\alpha} \sigma_{D}^{2}}{P_{S}} \beta+\frac{\operatorname{erf}(\sqrt{r}) \pi^{2} \lambda}{2} \sqrt{\frac{d_{S D}^{\alpha} P_{k D}}{P_{S}}} \beta\right)\right], \quad \beta>0 .
$$

where $r$ is radius of finite area with the intensity of each interferer $\lambda$, and $d_{S D}$ is the distance between source and destination.

From the outage probability (30), the spatial intensity of transmitting nodes $q_{D T}^{-1}(\varepsilon)$ can be obtained as

$$
q_{D T}^{-1}(\varepsilon)=\frac{1}{\pi^{2} \sqrt{\beta} \operatorname{erf}(\sqrt{r})} \sqrt{\frac{P_{S}}{d_{S D}^{\alpha} P_{i D}}}\left[\ln (1-\varepsilon)^{-1}-\left(\frac{d_{S D}^{\alpha} \sigma_{D}^{2}}{P_{S}}\right) \beta\right] .
$$

From the above equation, unlike transmission capacity in Weber et al. [13], we can find that $q^{-1}(\epsilon)$ can be a negative number due to thermal noise. Therefore, as density of transmitting nodes cannot be a negative number, transmission capacity with noise can be redefined as follows

$$
C_{D T}(\varepsilon)=\left\{\begin{array}{ll}
0, & \text { if } q^{-1}(\varepsilon) \leq 0 \\
R q^{-1}(\varepsilon)(1-\varepsilon), & \text { if } q^{-1}(\varepsilon)>0
\end{array} \quad\left(\mathrm{bps} / \mathrm{Hz} / \mathrm{m}^{2}\right) .\right.
$$

Finally, the transmission capacity of direct transmission with noise when $q^{-1}(\epsilon)>0$ can be written as

$$
C_{D T}(\varepsilon)=\frac{2 R(1-\varepsilon)}{\pi^{2} \sqrt{\beta} \operatorname{erd}(\sqrt{r})} \sqrt{\frac{P_{S}}{d_{S D}^{\alpha} P_{i D}}}\left[\ln (1-\varepsilon)^{-1}-\left(\frac{d_{S D}^{\alpha} \sigma_{D}^{2}}{P_{S}}\right) \beta\right] .
$$

\subsection{Dual-hop relaying}

Since we are concerned with the outage probability of the relay network after two-hop transmission, the transmission capacity of dual-hop relay is identical to that of single hop except for the target $\operatorname{SINR} \beta$.

Meanwhile, the transmission capacity of AF relaying cannot be obtained directly due to no closed-form of outage probability. Hence, the equivalent end-to-end SINR with Macdonald r.v. for AF strategy (24) can be written as

$$
\frac{X Y}{X+Y+1}<\frac{X Y}{X+Y}<\min [X, Y]
$$

where $\min [\mathrm{X}, \mathrm{Y}]$ is end-to-end SINR of DF relaying (14). As we focus on the information theoretical capacity, the fact that DF can be the upper bound of AF is 
established in it. Using this relation, we note that the transmission capacity of DF is the upper bound of AF relaying as follows

$$
C_{\mathrm{AF}}(\varepsilon)<C_{\mathrm{DF}}(\varepsilon)=C_{R}(\varepsilon)=\left\{\begin{array}{ll}
0, & \text { if } q^{-1}(\varepsilon) \leq 0 \\
R q^{-1}(\varepsilon)(1-\varepsilon), & \text { if } q^{-1}(\varepsilon)>0
\end{array} \quad\left(\mathrm{bps} / \mathrm{Hz} / \mathrm{m}^{2}\right)\right.
$$

where $\epsilon$ is the outage probability for the target $\operatorname{SINR} \beta$ $=2^{2 R}-1$ with spectral efficiency $R$. In this paper, we consider a single transmission capacity of dual-hop relay $C_{R}$ $(\epsilon)$ regardless of its protocols. From outage probability (16), we can obtain transmission capacity of dual-hop relay $C_{R}(\epsilon)$ as

$$
\begin{aligned}
C_{R}(\varepsilon)= & \frac{2 R(1-\varepsilon)}{\pi^{2} \sqrt{\beta} \operatorname{erf}(\sqrt{r})\left(\sqrt{\frac{d_{S R}^{\alpha} P_{i R}}{P_{S}}}+\sqrt{\frac{d_{R D}^{\alpha} P_{i D}}{P_{R}}}\right)} \\
& \times\left[\ln (1-\varepsilon)^{-1}-\left(\frac{d_{S R}^{\alpha} \sigma_{R}^{2}}{P_{S}}+\frac{d_{R D}^{\alpha} \sigma_{D}^{2}}{P_{R}}\right) \beta\right], \quad \text { for } q^{-1}(\varepsilon)>0
\end{aligned}
$$

where $\epsilon$ is outage probability of dual-hop relaying for target SINR $\beta$.

\section{Numerical and simulation results}

In this section, we present some numerical examples of the outage probability and transmission capacity for a dual-hop relaying with both interference and noise in a Poisson network. We consider Rayleigh fading channel and path loss exponent $\alpha=4$ to illustrate our analytic and simulated results.

\subsection{Outage probability}

Figures 2 and 3 show the analytic and simulated outage probability as a function of transmission power of a source and a relay $P_{S}=P_{R}$ for DF and AF strategies considering both noise and interference in a Poisson network, respectively. Both figures show that outage probability with end-to-end spectral efficiency $R=1$ bps $/ \mathrm{Hz}$ (target SINR $\beta=3$ ) and noise variance $\sigma_{R}^{2}=\sigma_{D}^{2}=1$ for various system parameters. Figures $2 \mathrm{a}$ and 3 a depict the outage probability as a function of transmission power with different intensity of interferers for DF and AF strategies, respectively. Both figures reveal significant decrease of outage performance and have an error floor with increasing intensity of interferes $\lambda$. Figure $2 b$ shows that outage probability of DF relaying with different distance pair $\left(d_{S R}, d_{R D}\right)=$ $(1.5,1.5),(1.5,2)$ and $(2,2)$. It is noted that the outage performance of dual-hop relaying is degraded by increasing their each hop distance $d_{S R}$ and $d_{R D}$, respectively. For AF relaying, increasing interference power is greatly influenced by outage probability performance in Figure 3b. In addition, we can see that the analysis agrees exactly with the simulation results from Figures 2 and 3.
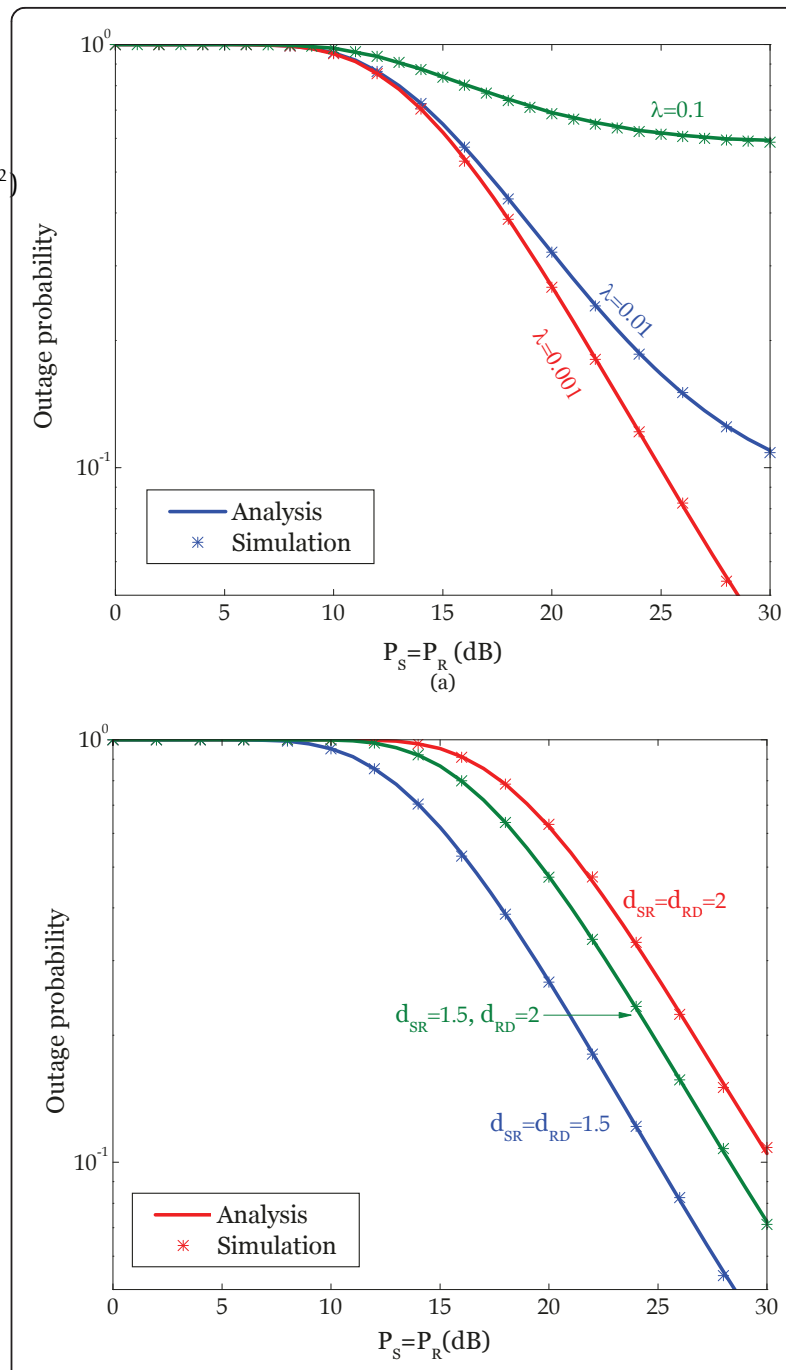

(b)

Figure 2 Outage probability as a function of $P_{S}=P_{R}$ for the DF relaying at the end-to-end spectral efficiency $R=1 \mathrm{bps} / \mathrm{Hz}$ in Rayleigh fading channel with path loss exponent $\alpha=4$, noise variance $\sigma_{R}^{2}=\sigma_{D}^{2}=1$ and interference power $P_{i R}=0.05 \times P_{S}$ $\left(P_{i R}=P_{i D}\right)$. (a) Different intensity of interferers $\lambda$ case when distance of each node $d_{S R}=d_{R D}=1.5$. (b) Different distance $d_{S R}$ and $d_{R D}$ node pair case when intensity of interferers $\lambda=0.001$.

\subsection{Transmission capacity}

We assume that the destination node is located $d_{S D}=\sqrt{3} \times d_{S R}$ away from source node for a fair comparison, when $d_{S R}$ is the same as $d_{R D}$ in transmission capacity. Figure 4 shows the spatial intensity of attempted transmission associated with outage probability $q^{-1}(\epsilon)$ as a function of outage probability with noise variance $\sigma_{R}^{2}=\sigma_{D}^{2}=1$ and interference power $P_{i R}=P_{S} \times 0.05$ at the end-to-end spectral efficiency $R=1 \mathrm{bps} / \mathrm{Hz}$. We compare $q^{-1}(\epsilon)$ of a dualhop relaying with direct transmission for different transmission power $P_{S}=P_{R}=15$ and $20 \mathrm{~dB}$. As we have seen 


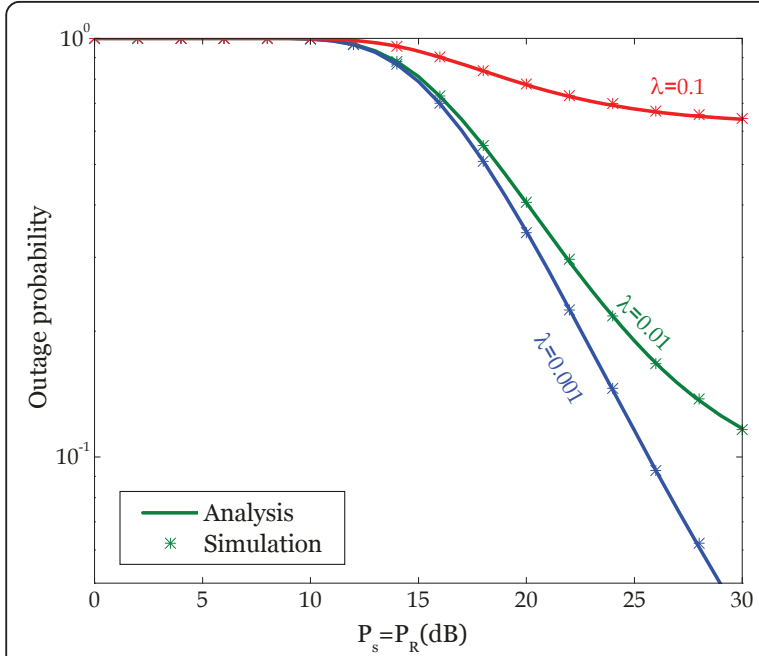

(a)

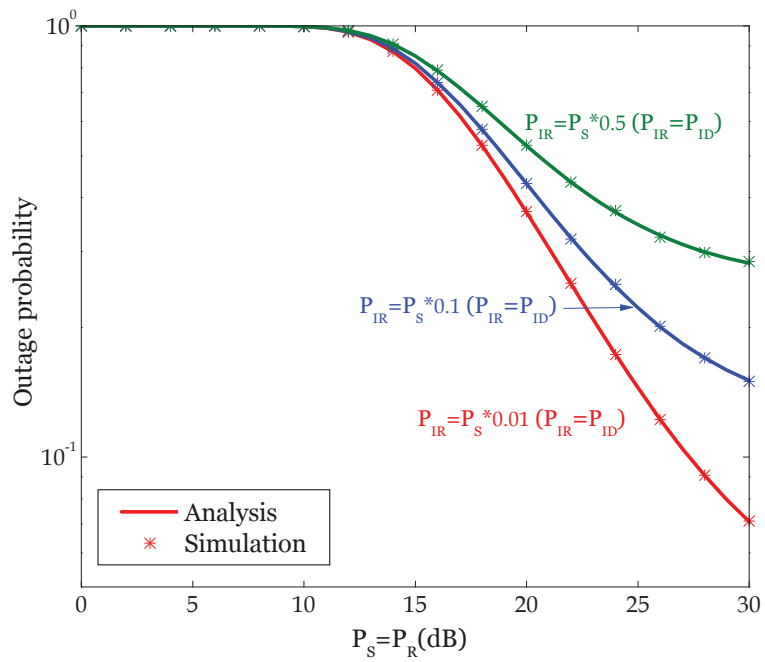

(b)

Figure 3 Outage probability as a function of $P_{S}=P_{R}$ for the AF relaying at the end-to-end spectral efficiency $R=1 \mathrm{bps} / \mathrm{Hz}$ in Rayleigh fading channel with path loss exponent $\alpha=4$, noise variance $\sigma_{R}^{2}=\sigma_{D}^{2}=1$ and distance $d_{S R}=d_{R D}=1.5$. (a) Different intensity of interferers $\lambda$ case when interference power $P_{i R}$ $=0.05 \times P_{S}\left(P_{i R}=P_{i D}\right)$. (b) Different interference power $P_{i R}$ and $P_{i D}$ case when intensity of interferers $\lambda=0.01$.

from (32) and (34), this figure shows that $q^{-1}(\epsilon)$ can be zero in relatively low-outage probability regime due to thermal noise. On the contrary, in high-outage probability regime, the curves in Figure 4 have a crossing point at $P_{S}$ $=P r=20 \mathrm{~dB}$ due to a loosen outage probability constraint, and it means that the advantage of using relay can be decreasing at communication system which permits low QoS. However, the dual-hop relaying has more transmitting nodes to satisfy outage probability than does direct transmission at most of outage probability regime.

Transmission capacity as a function of outage probability with noise variance $\sigma_{R}^{2}=\sigma_{D}^{2}=1$ and interference power $P_{i R}=P_{S} \times 0.05$ at the end-to-end spectral efficiency $R=1 \mathrm{bps} / \mathrm{Hz}$ is plotted in Figures 5 and 6. Figure 5 also compares direct transmission in terms of transmission capacity for different distance $d_{S R}=d_{R D}=0.5$, 1.5, and 2. Likewise Figure 4, the transmission capacity of dual-hop relay performs better than that of direct transmission, especially in the relatively low outage probability regime and long distance. That is, using a dual-hop relay has more successful transmission nodes to satisfy outage probability and data rate. Conversely, the transmission capacity of dual-hop relaying is similar or lower than direct transmission in high-outage probability regime and short distance. But, since we utilize a relay to transmit over long distance and concern lowoutage probability regime, the dual-hop relaying can be still efficient from transmission capacity point of view. Figures 4 and 5 reveal significant gain of dual-hop relaying compared to direct transmission in terms of density of attempted transmission and transmission capacity.

Finally, Figure 6 shows that transmission capacity of dual-hop relay at different transmission power $P_{S}=\operatorname{Pr}=$ 20 and $25 \mathrm{~dB}$ and distance $d_{S R}=d_{R D}=1.5$ and 2. Figure 6 also shows that the existing region $\left(q^{-1}(\epsilon)>0\right)$ of transmission capacity of a dual-hop relay is growing with increasing transmitting power $P_{S}=P r$ and decreasing distance $d_{S R}=d_{R D}$. This is due to the transmission capacity with thermal noise can be zero for small

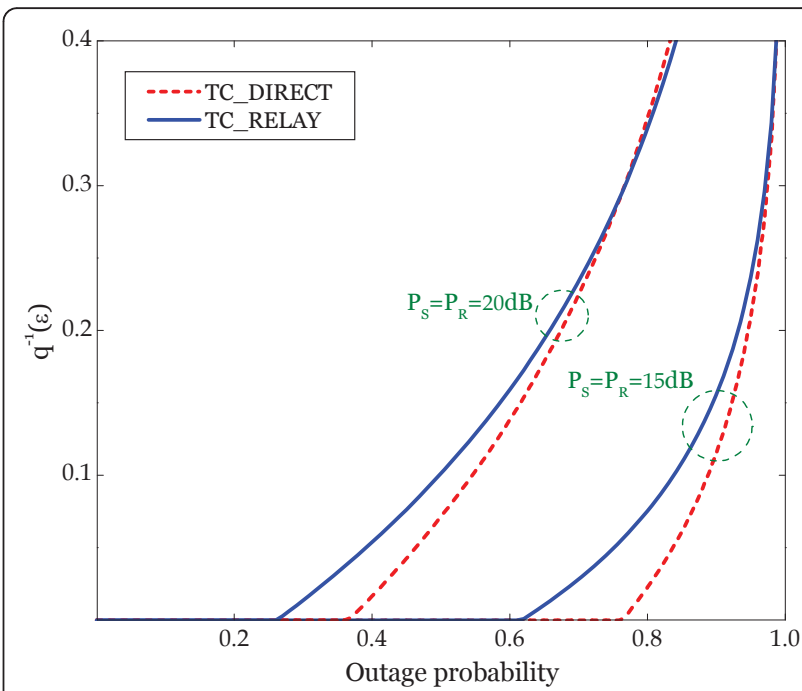

Figure 4 The spatial intensity of attempted transmission associated with outage probability $q^{-1}(\epsilon)$ as a function of outage probability $\epsilon$ for the dual-hop relaying at the end-toend spectral efficiency $R=1 \mathrm{bps} / \mathrm{Hz}$ in Rayleigh fading channel with path loss exponent $\alpha=4$ and noise variance $\sigma_{R}^{2}=\sigma_{D}^{2}=$ 1 and interference power $P_{i R}=0.05 \times P_{S}\left(P_{i R}=P_{i D}\right)$. Dual-hop relay is compared with direct transmission for different transmitting power $P_{S}=P_{R}=15$ and $20 \mathrm{~dB}$ when distance between source and destination $d_{S D}=d_{S R} \times \sqrt{3}$. 


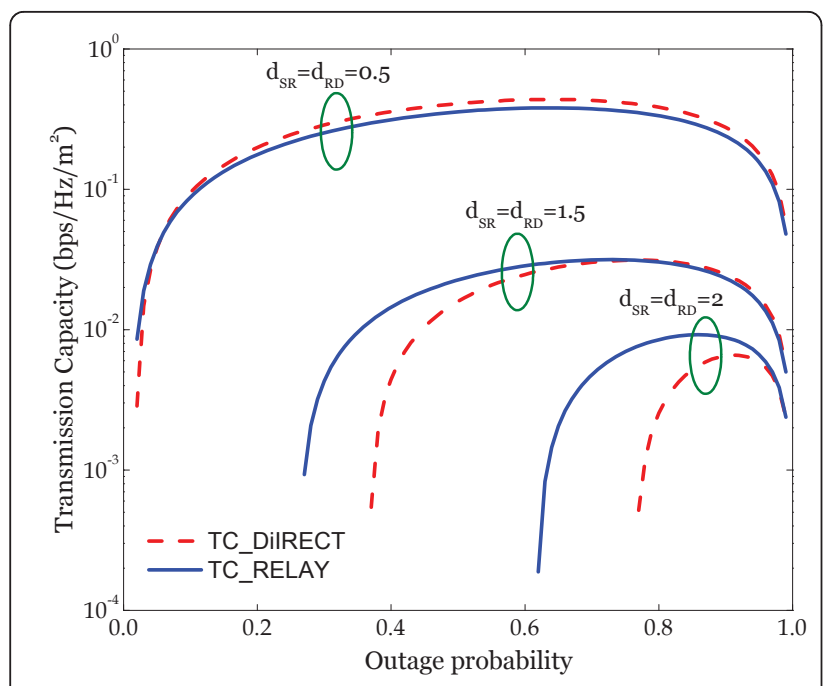

Figure 5 Transmission capacity as a function of outage probability $\epsilon$ for the dual-hop relaying at the end-to-end spectral efficiency $R=1 \mathrm{bps} / \mathrm{Hz}$ in Rayleigh fading channel with path loss exponent $\alpha=4$, noise variance $\sigma_{R}^{2}=\sigma_{D}^{2}=1$, transmitting power $P_{S}=P_{R}=25 \mathrm{~dB}$, and interference power $P_{i R}=0.05 \times P_{S}\left(P_{i R}=P_{i D}\right)$. Dual-hop relay is compared with direct transmission for different distance $d_{S R}=d_{R D}=0.5,1.5$ and 2 when distance between source and destination $d_{S D}=d_{S R} \times \sqrt{3}$.

transmission power and large distance. In common, the maximum transmission capacity is achieved at a specific outage probability in Figures 5 and 6. It is because that the transmission capacity consists of spatial density $q^{-1}$ $(\epsilon)$ and success probability $(1-\epsilon)$ from the definition $C$

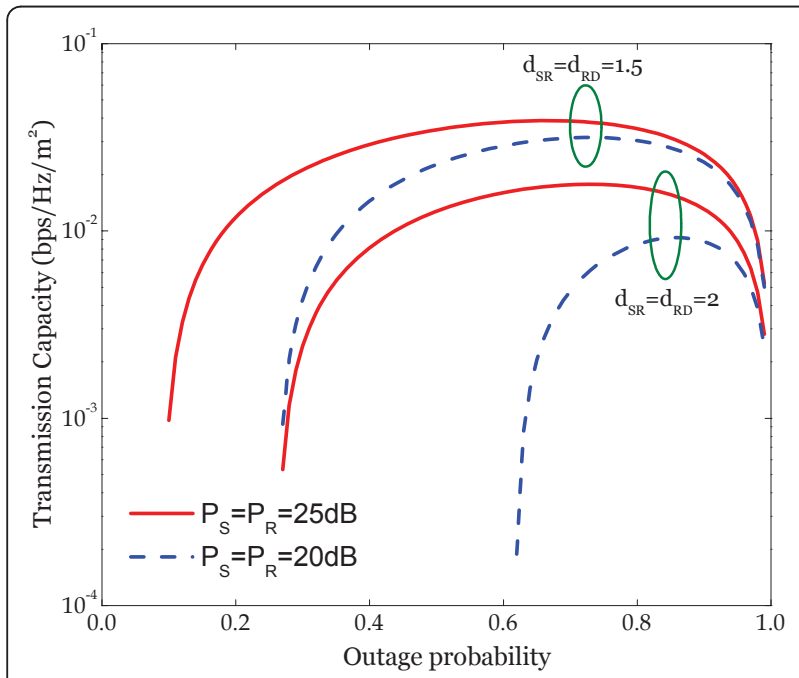

Figure 6 Transmission capacity as a function of outage probability $\epsilon$ for the dual-hop relaying at the end-to-end spectral efficiency $R=1 \mathrm{bps} / \mathrm{Hz}$ in Rayleigh fading channel with path loss exponent $\alpha=4$ and noise variance $\sigma_{R}^{2}=\sigma_{D}^{2}=$ 1 and interference power $P_{i R}=0.05 \times P_{S}\left(P_{i R}=P_{i D}\right)$ and $d_{S R}=$ $d_{R D}=1.5$ and 2 when $P_{S}=P_{R}=20$ and $25 \mathrm{~dB}$.
$(\epsilon)=R q^{-1}(\epsilon)(1-\epsilon)$. In other words, as we have seen from Figure $4, q^{-1}(\epsilon)$ increases consistently due to less outage constraint, but the success probability $(1-\epsilon)$ goes to zero as the outage probability increases. Therefore, the transmission capacity decreases due to the success probability in high-outage probability regime and has maximum value at a specific outage probability.

\section{Conclusion}

This paper considers a dual-hop relaying in the presence of both noise and interference simultaneously, allowing a Poisson interference model. The outage probability of DF and AF strategies have been derived, especially we verified that the end-to-end SINR of AF relaying has Macdonald r.v's. The analytic and simulated results showed that outage probability of dual-hop relay had an error floor in high density of transmitting nodes and performance was greatly influenced by distance and interference power. Furthermore, we consider a metric of decentralized wireless network, called transmission capacity, for dual-hop relay with AF and DF strategies. We redefine the transmission capacity, because spatial intensity of attempted transmission associated with outage probability $q^{-1}(\epsilon)$ can be negative number due to thermal noise. Hence, we note that the existing region $\left(q^{-1}(\epsilon)>0\right)$ of transmission capacity of dual-hop relay is growing with increasing transmission power. Our results reveal that transmission capacity of a dual-hop relay considering both noise and interference has better performance than direct transmission in a wireless ad hoc network.

\section{Appendix}

\section{A Proof of Theorem 1}

The CDF of end-to-end SINR at the relay $\mathrm{X} \sim \gamma_{R}^{\mathrm{DF}}$ is given by

$$
\begin{aligned}
F_{X}(x)= & \bullet\left\{\frac{\mathrm{P}}{\mathrm{Q}+\sigma_{R}^{2}}<x\right\} \\
= & \int_{0}^{\infty} p_{Q}(q) \int_{0}^{x\left(q+\sigma_{R}^{2}\right)} p_{P}(p) \mathrm{d} p \mathrm{~d} q \\
= & \int_{0}^{\infty} p_{Q}(q)\left[1-\exp \left(-\frac{d_{S R}^{\alpha}}{P_{S}} x\left(q+\sigma_{R}^{2}\right)\right)\right] \mathrm{d} q \\
= & 1-\int_{0}^{\infty} \exp \left(-\frac{d_{S R}^{\alpha}}{P_{S}} x\left(q+\sigma_{R}^{2}\right)\right) \frac{\operatorname{erf}\left(\sqrt{r_{1}}\right) \lambda_{1} P_{i R}^{\frac{1}{2}}}{4} \\
& \times\left(\frac{\pi}{q}\right)^{3 / 2} \exp \left(-\frac{\left[\operatorname{erf}\left(\sqrt{r_{1}}\right)\right]^{2} \pi^{4} \lambda_{1}^{2} P_{i R}}{16 q}\right) \mathrm{d} q \\
= & 1-\frac{\operatorname{erf}\left(\sqrt{R_{1}}\right) \lambda_{1} P_{i R}^{\frac{1}{2}} \pi^{3 / 2}}{4} \exp \left(-\frac{d_{S R}^{\alpha}}{P_{S}} x \sigma_{R}^{2}\right) \\
& \times \int_{A}^{\infty}\left(\frac{1}{q}\right)^{\frac{3}{2}} \exp \left[-\left(\frac{d_{S R}^{\alpha}}{P_{S}} x q+\frac{\left[\operatorname{erf}\left(\sqrt{r_{1}}\right)\right]^{2} \pi^{4} \lambda_{1}^{2} P_{i R}}{16 q}\right)\right] \mathrm{d} q
\end{aligned}
$$


$A$ can be changed to closed-form using following equation in Gradshteyn and Ryzhik [18]

$$
\int_{0}^{\infty} x^{-n-\frac{1}{2}} \mathrm{e}^{-(p x+q / x)} \mathrm{d} x=(-1)^{n} \sqrt{\frac{\pi}{p}} \frac{\partial^{n}}{\partial q} \mathrm{e}^{-2} \sqrt{p q}
$$

In this case, we define $n=1$, $p=\frac{d_{S R}^{\alpha} x}{P_{S}}, q=\frac{\left[\operatorname{erf}\left(\sqrt{R_{1}}\right)\right]^{2} \pi^{4} \lambda_{1}^{2} P_{i R}}{16}$. Then, the CDF of $\mathrm{X}$ can be written as

$$
F_{X}(x)=1-\exp \left[-\left(\frac{d_{S R}^{\alpha} \sigma_{R}^{2}}{P_{S}} x+\frac{\operatorname{erf}\left(\sqrt{r_{1}}\right) \pi^{2} \lambda_{1}}{2} \sqrt{\frac{d_{S R}^{\alpha} P_{i R}}{P_{S}} x}\right)\right], \quad x \geq 0
$$

Finally, we can obtain $C D F$ and $P D F$ of $X$ in Theorem 1.

\section{B Proof of Theorem 2}

From the $P D F$ and $C D F$ of $\mathrm{X}$ and $\mathrm{Y}$, we can compute outage probability of AF relaying as

$$
\begin{aligned}
& P_{\text {out }}^{\text {A }}(\beta)=\cdot\left\{\frac{\mathrm{XY}}{\mathrm{X}+\mathrm{Y}+1}<\beta\right\} \\
& =F_{\mathrm{Y}}(z)+\int^{\infty} F_{\mathrm{X}}\left(\frac{z(y+1)}{\gamma-z}\right) p_{\mathrm{Y}}(y) \mathrm{d} y \\
& =1-\int_{z}^{\infty} \exp \left[-\left(A\left(\frac{z(y+1)}{\gamma-z}\right)+C \sqrt{\left(\frac{z(y+1)}{\gamma-z}\right)}\right)\right] p_{\mathrm{Y}}(\gamma) \mathrm{d} y \\
& =1-\int_{z}^{\infty}\left(B+\frac{D}{2 \sqrt{y}}\right) \exp \left[-\left(A\left(\frac{z(y+1)}{y-z}\right)+B y+C \sqrt{\frac{z(y+1)}{y-z}}+D \sqrt{y}\right)\right] \mathrm{d} y \\
& =1-B \int_{2}^{\infty} \exp \left[-\left(A\left(\frac{z(y+1)}{y-z}\right)+B y+C \sqrt{\frac{z(y+1)}{y-z}+D \sqrt{y}}\right)\right] \mathrm{d} y \\
& -\frac{D}{2} \int_{z}^{\infty} \frac{1}{\sqrt{y}} \exp \left[-\left(A\left(\frac{z(y+1)}{y-z}\right)+B y+C \sqrt{\frac{z(y+1)}{y-z}}+D \sqrt{y}\right)\right] \mathrm{d} y
\end{aligned}
$$

where

$$
\begin{aligned}
& \beta=2^{2 R}-1, \quad A=\frac{d_{S R}^{\alpha} \sigma_{R}^{2}}{P_{S}}, \quad B=\frac{d_{R D}^{\alpha} \sigma_{D}^{2}}{P_{R}} \\
& C=\frac{\operatorname{erf}\left(\sqrt{r_{1}}\right) \pi^{2} \lambda_{1}}{2} \sqrt{\frac{d_{S R}^{\alpha} P_{i R}}{P_{S}}}, \quad D=\frac{\operatorname{erf}\left(\sqrt{r_{2}}\right) \pi^{2} \lambda_{2}}{2} \sqrt{\frac{d_{R D}^{\alpha} P_{i D}}{P_{R}}} .
\end{aligned}
$$

Using $y-z=u$, we can obtain outage probability of AF relaying in Theorem 2 .

\section{Acknowledgements}

This research was supported by the KCC (Korea Communications Commission), Korea, under the "Development of highly efficient transmission technology for the next generation terrestrial 3D HDTV" support program supervised by the KCA (Korea Communications Agency) (KCA-2011-1091202002).

\section{Author details}

'School of Electrical Engineering, Korea University, 5-1 Anam-dong, Sungbukgu, Seoul, Republic of Korea ${ }^{2}$ Department of Electronics and Radio Engineering, Kyung Hee University, 1732 Deogyeong-daero, Giheung-gu, Yongin-si, Gyeonggi-do 446-701, Republic of Korea ${ }^{3}$ Department of Electronics Engineering, Konkuk University, Seoul 143-701, Republic of Korea

\section{Competing interests}

The authors declare that they have no competing interests.

Received: 1 May 2011 Accepted: 22 February 2012

Published: 22 February 2012

\section{References}

1. A Sendonaris, E Erkip, B Aazhang, User cooperation diversity—part I: system description. IEEE Trans Commun. 51(11), 1927-1938 (2003). doi:10.1109/ TCOMM.2003.818096

2. IN Laneman, DNC Tse, GW Wornell, Cooperative diversity in wireless networks: efficient protocols and outage behavior. IEEE Trans Inform Theory. 50(12), 3062-3080 (2004). doi:10.1109/TIT.2004.838089

3. A Bletsas, H Shin, MZ Win, Cooperative communications with outageoptimal opportunistic relaying. IEEE Trans Wirel Commun. 6(9), 3450-3460 (2007)

4. B Barua, HQ Ngo, H Shin, On the sep of cooperative diversity with opportunitic relaying. Electon Lett. 12(10), 727-729 (2008)

5. I Krikidis, SM John, S Thompson, N Geortz, Max-min relay selection for legacy amplify-and-forward systems with interference. IEEE Trans Wirel Commun. 8(6), 3016-3027 (2009)

6. C Zhong, S Jin, K-K Wong, Dual-hop system with noisy relay and interference-limited destination. IEEE Trans Commun. 58(3), 764-768 (2010)

7. J Si, Z Li, Z Liu, Outage probability of opportunistic relaying in rayleigh fading channels with multiple interferers. IEEE Signal Process Lett. 17(5), 445-448 (2010)

8. P Gupta, PR Kumar, Transport capacity. IEEE Trans Inform Theory. 46(2), 388-404 (2000). doi:10.1109/18.825799

9. SP Weber, X Yang, JG Adrews, G de Veciana, Transmission capacity of wireless ad hoc networks with outage constraints. IEEE Trans Inform Theory. 51(12), 4091-4102 (2005). doi:10.1109/TIT.2005.858939

10. S Weber, JG Adrews, N Jindal, The effect of fading, channel inversion, and threshold scheduling on ad hoc networks. IEEE Trans Inform Theory. 53(11), 4127-4149 (2007)

11. AM Hunter, JG Adrews, S Weber, Capacity scaling of ad hoc networks with spatial diversity. IEEE Trans Wirel Commun. 7(12), 5058-5071 (2008)

12. K Huang, JG Adrews, J Robert, W Heath, D Guo, RA Berry, Spatial interference cancelation for mobile ad hoc networks. IEEE Trans Inform Theory, (2008) http://arxiv.org/abs/0807.1773

13. S Weber, JG Adrews, N Jindal, A tutorial on transmission capacity. IEEE Trans Inform Theory, (2009) http://arxiv.org/abs/0809.0016

14. R Vaze, KT Troung, S Weber, J Robert, W Heath, Two-way transmission capacity of wireless ad-hoc networks. IEEE Trans Inform Theory, (2010) http://arxiv.org/abs/1009.1460

15. JG Adrews, S Weber, M Kountouris, M Haenggi, Random access transport capacity. IEEE Trans Wirel Commun. 9(6), 2101-2111 (2010)

16. M Haenggi, RK Ganti, Interference in Large Wireless Networks (now - The essence of knowledge, Massachusetts, 2010)

17. D Daley, D Vere-Jones, An Introduction to the Theory of Point Processes (Springer, Berlin, 2003)

18. IS Gradshteyn, IM Ryzhik, Table of Integrals, Series, and Products, 6th edn. (Academic, San Diego, 2000)

\section{doi:10.1186/1687-1499-2012-58}

Cite this article as: Lee et al:: Transmission capacity for dual-hop relaying in wireless ad hoc networks. EURASIP Journal on Wireless Communications and Networking 2012 2012:58. 